ARTICLE

\title{
Full-Scale 3D Vibration Simulator for an Entire Nuclear Power Plant on the Simple Orchestration Application Framework
}

\author{
Guehee KIM*, Kohei NAKAJIMA, Naoya TESHIMA, \\ Takayuki TATEKAWA, Yoshio SUZUKI and Hiroshi TAKEMIYA
}

Japan Atomic Energy Agency, 6-9-3 Higashi-ueno, Taito-ku, Tokyo, 110-0015, Japan

\begin{abstract}
So far, we have developed Full-scale 3D Vibration Simulator for an Entire Nuclear Power Plant which is simulation platform to analyze seismic response of a whole digitalized nuclear power plant. In this work, we introduced pipelined data-transfer scenario in which boundary condition data between plant's components are transferred each time step from large components to small components while all components simulations are run in parallel. We developed grid-enabled application to perform this simulation more efficiently using various computing resources. In the development, we confronted two problems; first, pre-existing grid technology still does not sufficiently support to realize pipelined data-transfer and second, to realize long time simulation by the scenario, functionality enabling simulation restarting to be automated is necessary. As solution of these problems, we used Simple Orchestration Application Framework (SOAF) which enabled pipelined data-transfer to be realized in easy and simple way and simulation restarting to be automated. Using the SOAF, we developed grid-enabled application and succeeded seismic analysis simulation of a test reactor in pipelined data-transfer scenario for about a week. In this paper, the details of all of this work will be presented.
\end{abstract}

KEYWORDS: nuclear power plant, simulation, seismic analysis, grid

\section{Introduction}

In Japan of earthquake-prone country, earthquake resistance design and dynamic analysis of nuclear power plants (NPPs) are very important issues. Japan has over 50 NPPs and over 10 reactors among them are aging as more than 30 years old. Further, the Great Hanshin Earthquake which devastated the city of Kobe in 1995 and Chūetsu offshore earthquake in 2007 have heightened people's concern about the safety of NPPs. Thus, keeping of safe operation of NPPs becomes all the more important task, which contributes to stable atomic energy supply making people feel reliable.

"Center for Computational Science and e-Systems of Japan Atomic Energy Agency (CCSE/JAEA)" has performed R\&Ds to satisfy the above need and developed "Full-scale 3D Vibration Simulator for an Entire Nuclear Power Plant (hereafter, 3D VS)". ${ }^{1,2}$ 3D VS is a full-fledged simulation platform for seismic response analysis for a whole NPP, the concept of which is presented in Fig. 1. Since the 3D VS treats a whole digitalized NPP as an assembly structure composed of its digitalized parts, it is possible to elucidate physical phenomena between NPP parts in detail. Clarifying seismic performance of a whole NPP structure, we aim to help soundness evaluation of the existing NPPs and support desirable earthquake resistance design of new NPPs and upgrade of their design concepts.

Generally, full-fledged simulation of an NPP using the 3D VS needs high computing power and memory capacity that a present single supercomputer can not sufficiently supply.

*Corresponding author, E-mail:kim.guehee@jaea.go.jp

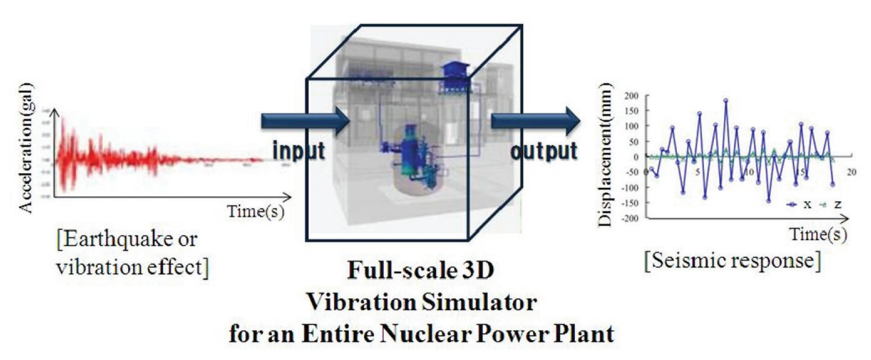

Fig. 1 "Full-scale 3D Vibration Simulator for an Entire Nuclear Power Plant" is full-fledged simulation platform for seismic response analysis for a whole digitalized nuclear power plant.

Furthermore, it is expected that, in future, simulation model and result data will reach petascale data so that we will need storage or database system which can manage the large data effectively and efficiently. For this reason, R\&D of parallel and distributed computing and data management technologies is very important issue. To address this issue, grid technology ${ }^{3,4)}$ which concerns about coupling various supercomputers geographically dispersed is an inevitable mean and in this context, we have developed grid-enabled application of the 3D VS from which an NPP model is deployed on various grid computing resources by plant component units.

In this work, we introduce pipelined data-transfer scenario by which all components are concurrently simulated transferring boundary condition data from large components to small components per each time step. In realizing the introduced scenario in the previous grid-enabled application, however, we confronted two problems: first problem is that 


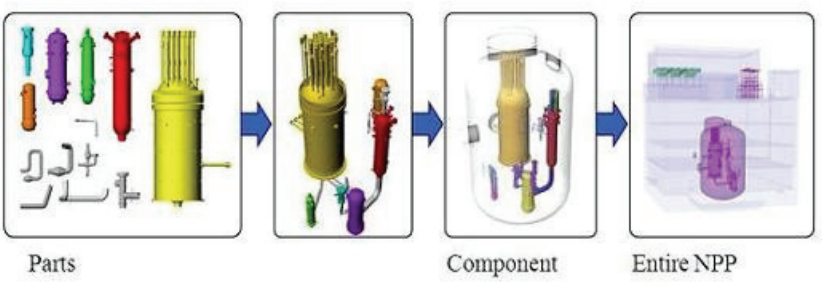

Fig. 2 In 3D VS, an NPP is considered of assembly structure composed of parts and components in finite element method model.

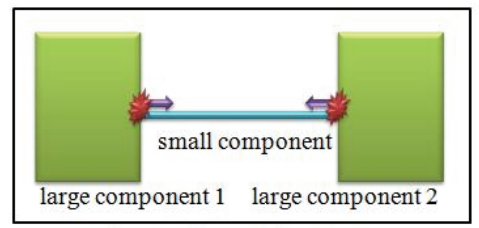

One-direction physical effect

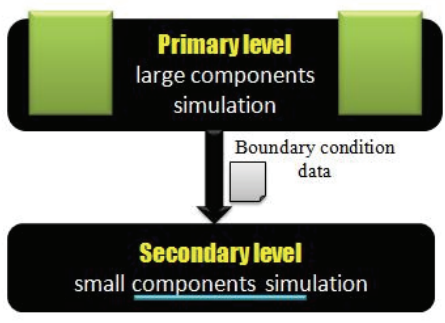

Hierarchical model

Fig. 3 In 3D VS, considering one-direction physical effect, all components are grouped into primary level of large components or secondary level of small components.

the previous application does not support data-transfer during jobs running and use of pre-existing grid technology is not efficient because its implementation is not sufficiently user-friend and needs an amount of simulation source codes modification. Second problem is to make simulation restarting automated for long time simulation. To clear these problems, we used "Simple Application Orchestration Framework (SOAF)" developed by CCSE/JAEA and upgraded previous grid-enabled application. To confirm the effectiveness of the SOAF, we performed seismic response analysis of "High Temperature engineering Test Reactor (HTTR)" at O-arai R\&D center of JAEA. In Section II, we explain 3D VS and discuss the above two problems in more detail. In Section III, we describe SOAF functionalities and its implementation in the previous grid-enabled application. In Section IV, simulation of HTTR using the application will be explained. In Section V, we summarize our work.

\section{Full-Scale 3D Vibration Simulator for an Entire Nuclear Power Plant}

\section{Hierarchical Methodology}

Distinguished point of $3 \mathrm{D}$ VS is that a whole digitalized NPP is divided by component units as shown in Fig. 2, where each component is an assembly structure with a number of NPP parts prepared in mesh model of finite elements method. Components are individually analyzed by 3D VS

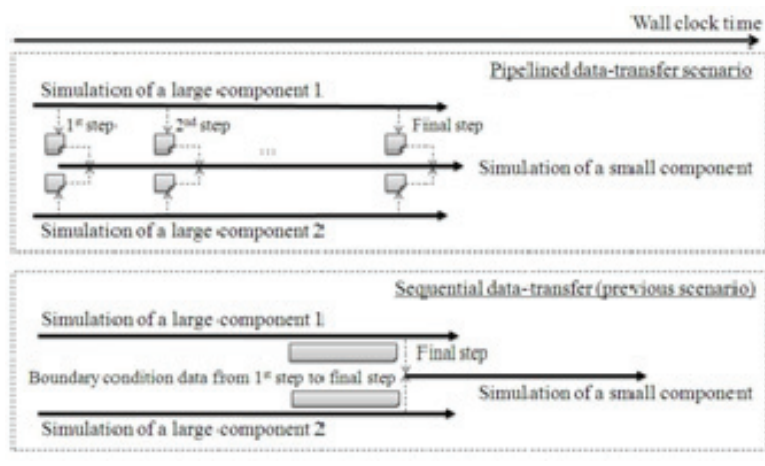

Fig. 4 Schematic view of pipelined data-transfer scenario and sequential data-transfer scenario in the case of component model shown in Fig. 3

solver and mutual vibration effects between parts are reflected by data exchange at parts boundaries per each simulation time step.

Large-mass components of NPPs, for example, reactor pressure vessel and cooling systems are interconnected by small-mass components such as piping systems. In theoretical study, it was shown that physical effects from small components to large components could be neglected compared to that the vice versa. ${ }^{5)}$ Based on this property, we introduced hierarchical manner shown in Fig. 3 by which we grouped large components at primary level and small components at secondary level and used boundary condition data obtained from simulation at primary level as input data to simulate secondary level.

\section{Pipelined Data-Transfer Method for Efficient Reali- zation of Hierarchical Methodology}

We introduce pipelined data-transfer scenario in which boundary condition data are transmitted per each time step from the primary level to the secondary level. Pipelined data-transfer scenario makes the whole simulation more efficient than the previous sequential data-transfer scenario. Schematic views of these two scenarios are presented in Fig. 4. In the sequential data-transfer scenario boundary condition data are accumulated during simulations at primary level and, after all of the simulations are completed, the data are used as input data with the start of simulations at secondary level. In general, for seismic response analysis for 20 seconds time-scale under earthquake, the number of demanded simulation time steps is about 1,000 . Wallclock time to simulate one time step for a piping system is usually a few ten minutes using present general supercomputers, which means that totally saved wallclock time by pipelined data-transfer scenario is roughly estimated at a few hundred hours which is necessary to simulate piping systems 1,000 time steps.

\section{Grid-Enabled Application of Full-Scale 3D Vi- bration Simulator}

1. Grid-Enabled Functionalities to Enable Long-Time Simulation by Pipelined Data-Transfer Scenario

An entire NPP is composed of parts from 100 thousands 


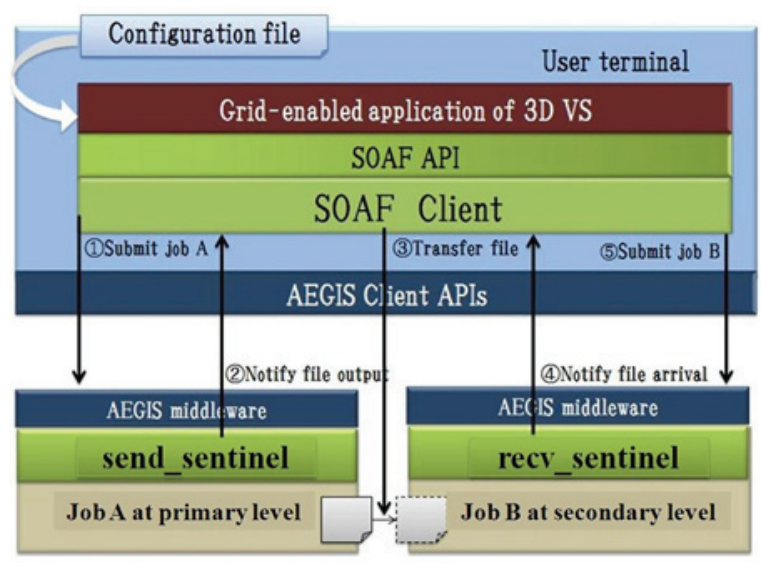

Fig. 5 System view adopting SOAF in grid-enabled application of 3D VS: (1) (5) is procedure of jobs submission and file transfer through collaboration between SOAF client, send_sentinel, and recv_sentinel.

to 10 millions so that generally, it is impossible to perform full-scale simulation on a single supercomputer. As analysis precision will become more and more accurate, prepared model data will be terabyte-scale size and we will need teraor peta- scale for computing performance, memory, and storage. In this respect, use of grid resources is desirable solution by deploying components on various grid computing resources. So far, we have developed grid-enabled application for 3D VS using client APIs (Application Program Interfaces) of grid environment of JAEA, "Atomic Energy Grid InfraStructure (AEGIS)" and have performed seismic analysis of HTTR. ${ }^{6-9)}$

By introducing the pipelined data-transfer scenario, we needed to enhance the previous grid-enabled application. In the enhancement, functionalities that should be newly added and problems involving them were as follows.

(1) Functionality that Transfers Files While Jobs Are Running

In the previous grid-enabled application order of files transfer and jobs execution can be only sequentially defined and files transfer during jobs are running is not supported. Use of pre-existing grid technology such as grid-enabled MPI or GridRPC might be a solution for the necessary functionality. Use of them, however, needs rewriting considerable parts of simulation source codes and, also demands expert-level understanding of the technology, which is time-consuming and hard work for code developers who are not grid experts. Furthermore, considering that the simulation code will be continuously upgraded with more advanced analytical functions, it seems that the pre-existing technology is not desirable and efficient choice.

(2) Functionality that Automatically Submits Jobs to Restart Simulation

To get physically meaningful simulation results on general supercomputers, long-time simulation more than a few weeks is necessary. Since job's allowed running time on batch queue system of supercomputers is limited to a few hours each submission, jobs are demanded to be resubmitted

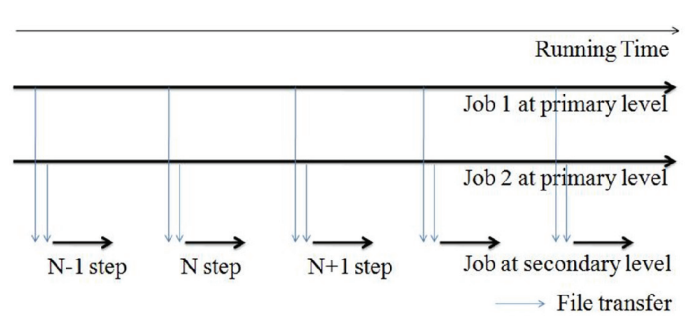

Fig. 6 Schematic view of "one time step execution" strategy of jobs at secondary level

a number of times to continue simulation just in the case of even one component simulation. In pipelined data-transfer scenario by which jobs of different components are concurrently executed, it is not realistic for users to keep on monitoring and manually restarting all of these jobs also checking input files whenever resubmitting them.

\section{Simple Orchestration Application Framework}

To tackle with the above problems, we adopted SOAF developed by CCSE/JAEA. ${ }^{10)}$ SOAF aims at supporting grid user's definition of complex order of jobs execution and files flow which could not be simply defined sequentially. In SOAF, it is possible to transfer files independent with jobs status and also, definition of jobs flow is based on files flow information on which file should be transferred from which job to which another job. Except jobs which are executed with the start of simulation, job submission timing depends on the arrival timing of their input files to their job directories. Also, SOAF checks each batch queue job's status and resubmits a new job when a job is killed due to its time limit exceed.

We upgraded the previous grid-enabled application using SOAF as shown in Fig. 5. SOAF provides SOAF API to support development of grid-enabled application and does not need an amount of simulation source codes modification. Files flow information is prepared by users in configuration file which is general text file and handed over to SOAF API through grid-enabled application. SOAF is composed of a client tool, SOAF client and server scripts, "send_sentinel" and "recv_sentinel". SOAF client is responsible for files transfer and submission and resubmission of jobs which are done by collaboration with the server scripts. The "send_sentinel" monitors target files output from submitted jobs and notifies the output to the SOAF client. The "recv_sentinel" monitors arrival of target files and notifies the arrival to the SOAF client.

For the jobs at secondary level, we introduced "one time step execution" strategy in Fig. 6 by which each job at secondary level is submitted after all of its input files per each time step arrive and is executed only one time step, which is repeated whenever next time step input files arrive until final step. By this strategy, simulations at secondary level can be performed without idle time which takes from a few minutes to a few hours per each time step just waiting files from the primary level. 

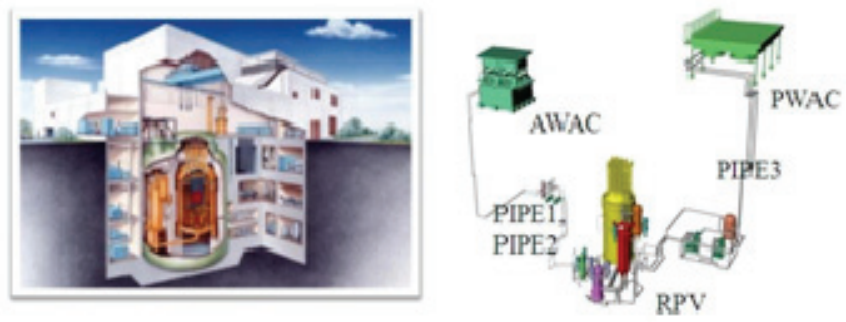

Fig. 7 Bird's-eye view of HTTR building (left side) and its digitalized model (right side) which is composed of six components

Table 1 Data size and parallelism of each component model

\begin{tabular}{cccc}
\hline $\begin{array}{c}\text { Hierarchical } \\
\text { level }\end{array}$ & $\begin{array}{c}\text { Component } \\
\text { name }\end{array}$ & $\begin{array}{c}\text { Model data size } \\
(\mathrm{GB})\end{array}$ & Parallelism \\
\hline Primary & RPV & 16.2 & 512 \\
& PWAC & 5.9 & 256 \\
& AWAC & 1.4 & 128 \\
Secondary & PIPE1 & 0.2 & 64 \\
& PIPE2 & 0.8 & 64 \\
& PIPE3 & 1.7 & 128 \\
\hline
\end{tabular}

\section{Experiment}

\section{Model}

Using the upgraded grid-enabled application, we performed full-scale seismic response analysis of HTTR in pipelined data-transfer scenario. HTTR, bird's-eye view of which is shown in Fig. 7, is research facility for development of high temperature gas-cooled reactor technology and nuclear heat utilization technology. Digitalized model of HTTR as shown in Fig. 7 is composed of six components: "Reactor Pressure Vessel (RPV)", "Pressurized Water Air Cooler (PWAC)", and "Auxiliary Water Air Cooler (AWAC)" at primary level and three interconnecting piping systems at secondary level. Total degree of freedom is about 150 million and total model data size is about $26 \mathrm{~GB}$. Table 1 shows model data size and parallelism of each component. We performed 100 simulation time steps using El Centro earthquake data.

\section{Deployment on AEGIS}

We used 1,024 CPUs of six queue job classes on a massive parallel supercomputer, Altix $3700 \mathrm{Bx} 2$ and a PC cluster machine, Altix350 on JAEA Tokai site of AEGIS shown in Fig. 8. To simplify file transfer processes, we placed TSS (Time Sharing System) programs between primary level and secondary level so that dozens of boundary condition data files from the primary level per each time step were integrated into one file by the TSS programs as shown in Fig. 9.

\section{Result}

During the whole simulation which took about 160 hours, all of data files were transferred to their target directories in pipelined scenario. Since there were the TSS programs, pipelined data-transfer was done through two stages: from primary level to TSS level and from the TSS level to secondary level as shown in Fig. 10. Immediate

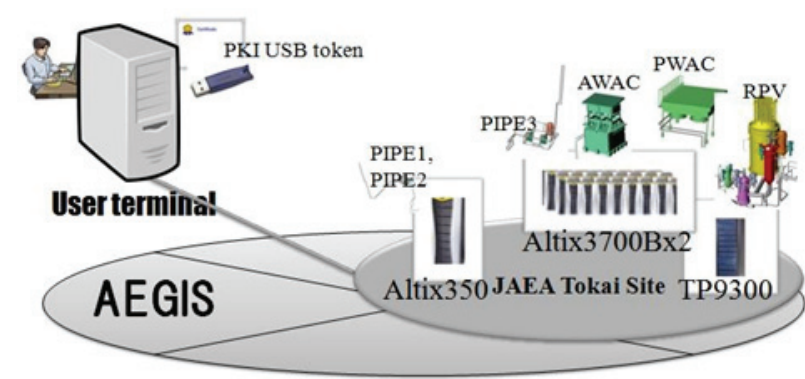

Fig. 8 Deployment view of six components of HTTR on computing resources on JAEA Tokai site of AEGIS

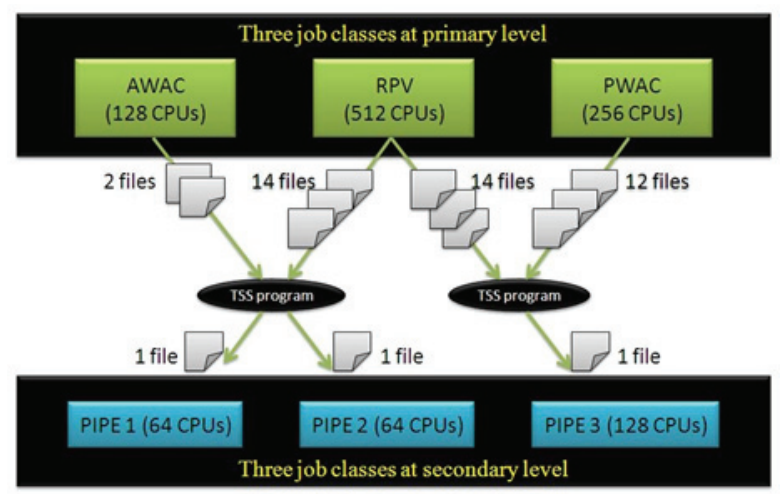

Fig. 9 Files transfer view per each time step from three job classes at primary level to three job classes at secondary level via TSS programs

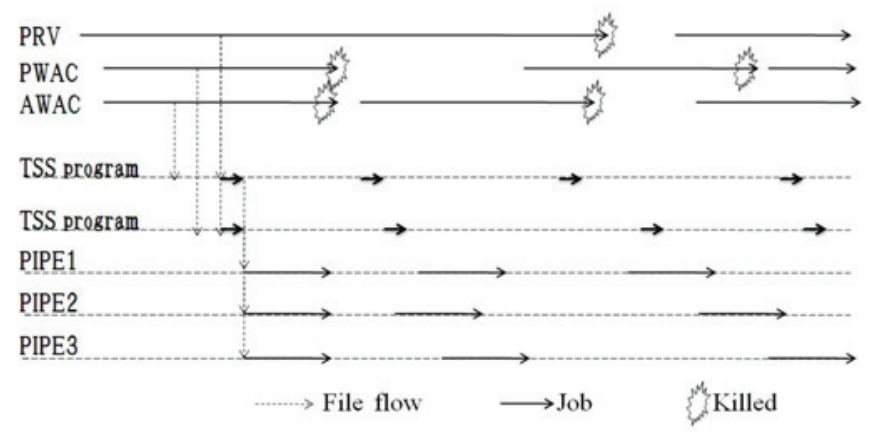

Fig. 10 Schematic view of jobs flow chart according to pipelined data-transfer scenario between six job classes and two TSS programs

Table 2 Number of resubmission time of jobs

\begin{tabular}{ccc}
\hline Component name & $\begin{array}{c}\text { Number of } \\
\text { resubmission time }\end{array}$ & $\begin{array}{c}\text { Time limit of job } \\
\text { classes (hours) }\end{array}$ \\
\hline RPV & 1 & 12 \\
PWAC & 3 & 3 \\
AWAC & 9 & 3 \\
\hline
\end{tabular}

resubmissions of jobs killed due to time limit exceed were done: one time for RPV, three times for PWAC, and 9 times for AWAC, where limit time of RPV job class was 12 hours and others were 3 hours (Table 2). In the case of jobs at secondary level, there was no resubmission since each job per each time step was terminated before reaching the time limit. 


\section{Summary}

CCSE/JAEA has performed R\&Ds of Full-scale 3D Vibration Simulator for an Entire Nuclear Power Plant which is seismic response analysis system for a whole digitalized NPP. Generally, full-fledged simulation of an NPP needs massive computing power and very huge storage and memory which a single supercomputer can not sufficiently sustain. To address this problem, we have developed grid-enabled application of 3D Vibration Simulator using client APIs of grid infrastructure AEGIS of JAEA. In simulation, an NPP model is deployed by component units on computing resources of AEGIS. 3D Vibration Simulator treats only physical effects from large components such as a reactor to small components such as interconnecting pipes. In this work, to reflect the one-directional physical effect in simulation, we introduced pipelined data-transfer scenario in which boundary condition data were transferred from the large components to small components per each time step, which made a whole simulation more efficient than the previous sequential scenario in which after completion of all simulations of large components, simulations of small components were begun. In introducing the pipelined data-transfer scenario, we confronted two challenges: first is how to realize the scenario on grid and second is automation of simulation restarting for long time simulation. To tackle with these challenges, we adopted Simple Orchestration Application Framework (SOAF) developed by CCSE/JAEA. SOAF has functionalities of files transfer and jobs submission depending on files flow and automatic resubmission of jobs. We implemented the SOAF in the previous grid-enabled application from which we performed full-scale seismic response analysis for High Temperature engineering Test Reactor at O-arai R\&D center of JAEA using six job classes of two supercomputers and succeeded simulation of almost a week.

\section{Acknowledgment}

We acknowledge O-arai R\&D center of JAEA for useful discussion and data provision.

\section{References}

1) M. Tani, N. Nakajima, A. Nishida, Y. Suzuki, H. Matsubara, F. Araya, N. Kushida, O. Hazama, K. Kawasaki, "A methodology of structural analysis for nuclear power plant size of assembly structure," Proc. of Mathematics \& Computation and Supercomputing in Nuclear Applications: the Joint International Topical Meeting, Apr. 15-19, 2007, California, USA (2007), [CD-ROM].

2) T. Yamada, F. Araya, "Construction of vibration table in an extended world for safety assessment of nuclear power plants", High Performance Computing on Vector Systems 2009, Springer, Heidelberg, 223-232 (2009).

3) I. Foster, C. Kesselman (eds.), The Grid: Blueprint for a New Computing Infrastructure, Morgan Kaufmann, California (1998).

4) I. Foster, C. Kesselman (eds.), The Grid 2: Blueprint for a New Computing Infrastructure, Morgan Kaufmann, California (2003).

5) A. Nishida, P. Liu, K. Kawaguchi, "Fundamental studies of wave-propagation properties of single layer lattice structures part1: impact experiments of cantilever beams by using impulse hammer," JSCE J. Struct. Eng., 46B, 175-179 (2000), [in Japanese].

6) Y. Suzuki, A. Nishida, F. Araya, N. Kushida, T. Akutsu, N. Teshima, K. Nakajima, M. Kondo, S. Hayashi, T. Aoyagi, N. Nakajima, "Development of three-dimensional virtual plant vibration simulator on grid computing environment ITBL-IS/AEGIS," J. Power Energy Syst., 3[1], 60-71 (2009).

7) G. Kim, Y. Suzuki, A. Nishida, H. Takemiya, "Development of APIs for desktop supercomputing," High Performance Computing on Vector Systems 2009, Springer, Heidelberg, 97-107 (2009).

8) Y. Suzuki, K. Nakajima, N. Kushida, C. Kino, T. Aoyagi, N. Nakajima, K. Iba, N. Hayashi, T. Ozeki, T. Totsuka, H. Nakanishi, Y. Nagayama, "Research and development of fusion grid infrastructure based on atomic energy grid infrastructure (AEGIS) ," Fusion Eng. Des., 83, 511-515 (2008).

9) G. Kim, Y. Suzuki, N. Teshima, A. Nishida, T. Yamada, F. Araya, H. Takemiya, N. Nakajima, M. Kondo, "A script generator API for the full-scale three-dimensional vibration simulation of an entire nuclear power plant within AEGIS," Proc. of the First International Conference on Parallel, Distributed and Grid Computing for Engineering, Pecs, Hungary, April 6-8, 2009, DOI:10.4203/ccp.90.22 (2009), [CD-ROM].

10) T. Tatekawa, K. Nakajima, N. Teshima, G. Kim, Y. Suzuki, H. Takemiya, N. Hayashi, K. Iba, "Simple Orchestration Application Framework to control "Burning Plasma Integrated Code"," Proc. of the Third International Joint Conference on Computational Science and Optimization, May 28-31, 2010, Huangshan, China, 2, 322-326 (2010). 